\title{
Process evaluation of a complex intervention to promote advance care planning in community-dwelling older persons (the STADPLAN study) - study protocol
}

Katharina Silies $^{1^{*}}$ (D), Rieke Schnakenberg ${ }^{2 *}$, Almuth Berg $^{3}$, Änne Kirchner $^{3}$, Henriette Langner $^{3}$, Juliane Köberlein-Neư ${ }^{4}$, Gabriele Meyer ${ }^{3}$, Falk Hoffmann ${ }^{2}$ and Sascha Köpke ${ }^{1,5}$

\begin{abstract}
Background: Process evaluation addresses the implementation, mechanisms of impact, and context of participants in complex interventions. The STADPLAN study assesses the effects of conversations on advance care planning (ACP) led by trained nurse facilitators. The complex intervention consists of several components that may lead to various changes in attitude and behavior regarding personal ACP activities. With the process evaluation, we aim to assess how changes were achieved in the STADPLAN intervention.

Methods: The planned process evaluation study will be conducted alongside a cluster-randomized controlled trial on ACP in home care services (HCS). Trained nurse facilitators will deliver the ACP intervention consisting of an information brochure and two ACP conversations. A logic model depicts the assumed change processes of the intervention: the educational program enables nurses to conduct ACP conversations with patients and their caregivers. Patients gain knowledge and reflect upon and engage in their own ACP. Caregivers better understand patients' wishes and feel reassured in their role as surrogates. Designation of a surrogate and communication on ACP are facilitated. We will assess the effects of the educational program with questionnaires and a focus group including all participating nurses. We will measure ACP engagement, and prevalence of advance directives in patients, and ask for their experiences with the intervention. We will conduct semi-structured interviews with caregivers about their expectations and experiences regarding ACP in general and the intervention. We will address context factors, e.g., basic characteristics of the HCS (such as ownership, number of clients, staff and qualification). Analysis will be based upon the logic model, integrating qualitative and quantitative data.

(Continued on next page)
\end{abstract}

\footnotetext{
*Correspondence: Katharina.silies@uksh.de; rieke.schnakenberg@uni-

oldenburg.de

${ }^{1}$ Institute for Social Medicine and Epidemiology, Nursing Research Unit,

University of Lübeck, Ratzeburger Allee 160, 23562 Lübeck, Germany

${ }^{2}$ Department for Health Services Research, Faculty of Medicine and Health

Sciences, Carl von Ossietzky University Oldenburg, Ammerländer Heerstraße

140, 26129 Oldenburg, Germany

Full list of author information is available at the end of the article
}

(c) The Author(s). 2020 Open Access This article is licensed under a Creative Commons Attribution 4.0 International License, which permits use, sharing, adaptation, distribution and reproduction in any medium or format, as long as you give appropriate credit to the original author(s) and the source, provide a link to the Creative Commons licence, and indicate if changes were made. The images or other third party material in this article are included in the article's Creative Commons licence, unless indicated otherwise in a credit line to the material. If material is not included in the article's Creative Commons licence and your intended use is not permitted by statutory regulation or exceeds the permitted use, you will need to obtain permission directly from the copyright holder. To view a copy of this licence, visit http://creativecommons.org/licenses/by/4.0/ The Creative Commons Public Domain Dedication waiver (http://creativecommons.org/publicdomain/zero/1.0/) applies to the data made available in this article, unless otherwise stated in a credit line to the data. 
(Continued from previous page)

Discussion: The comprehensive process evaluation will provide essential information on the feasibility of implementation strategies and the clinical relevance of a nurse-led ACP intervention in home care recipients and its generalizability and transferability to other settings.

Trial registration: German Clinical Trials Register: DRKS00016886. Registered retrospectively on June 4, 2019, first participant included on May 29, 2019.

Keywords: Process evaluation, Study protocol, Logic model, Complex intervention, Mixed methods, Home care setting, Ambulatory setting, Nursing, Advance care planning

\section{Contribution to the literature}

- The STADPLAN study is a large cluster-randomized controlled trial and one of the first trials on ACP in community dwellers. It will provide evidence on the effectiveness of an ACP intervention provided by nurses in home care

- The process evaluation as outlined in this paper will indicate how the ACP intervention is related to patient activation, $\mathrm{ACP}$ engagement, and surrogate designation and evaluate its feasibility in the home care setting

- The mixed methods approach based on the MRC framework for the development and evaluation of complex interventions will ensure high methodological rigor

- Results will support the implementation of ACP in the community setting

\section{Background}

\section{Advance care planning}

"Advance care planning is a process that supports adults at any age or stage of health in understanding and sharing their personal values, life goals, and preferences regarding future medical care. The goal of advance care planning is to help ensure that people receive medical care that is consistent with their values, goals, and preferences during serious and chronic illness" [1].

ACP can be documented in written form and relatives can be involved in the communication [2-4]. The need for ACP arose in relation to evolving medical treatment options and the increased possibilities to prolong life. To retain patients' autonomy despite unconsciousness or impaired decisional capacity has gained increasing weight. Legislation has been developed to protect patients' rights and to avoid medical paternalism [5-7]. As these cannot sufficiently address patients' needs, the concept of ACP evolved. Here, programs were developed that promoted reflection on personal values and communication with health professionals and within families $[8,9]$. In Germany, advance directives are legally binding by law since 2009 (German Advance Directives Act [Patientenverfügungsgesetz]) and ACP conversations costs can be covered by the statutory health insurance for people living in nursing homes or facilities providing integration assistance for disabled people since 2015 (Hospice and Palliative Care Act [Hospiz- und Palliativgesetz]). Despite such legislation, ACP and advance directives (AD) have not been sufficiently implemented [10]. Therefore, we will conduct a cluster-randomized controlled trial assessing a complex intervention aiming to promote ACP in community-dwelling older persons (the STADPLAN study: STudy on ADvance care PLANning in caredependent community dwelling older persons) [11].

\section{The STADPLAN study}

In this study, we will adapt an ACP program to the German home care setting, which has been successfully implemented in other countries and settings [12, 13], among these also German nursing homes [9]. We will include 16 home care services (HCS) both in the intervention and the control group, with a total of 960 participating HCS clients. Trained nurse facilitators of the HCS will deliver ACP. The primary endpoint is patient activation assessed by the German version of the Patient Activation Measure (PAM-13) [14, 15]. The PAM-13 is a valid and reliable instrument assessing the degree to which individuals take an active role in managing their own health, the corresponding health care and its consequences, and the extent to which individuals feel competent to fulfill that role.

We will measure secondary endpoints, such as proportion of persons with advance directives, hospitalization, and quality of life as well as depression and anxiety.

The study protocol of the cluster-randomized trial has been published [11]. This paper outlines the comprehensive process evaluation conducted alongside the trial.

\section{Process evaluation in complex interventions}

The complexity of interventions is determined by several dimensions, e.g., the number and interaction of components, the number and difficulty of behaviors required for delivering or receiving the intervention, or the number of groups or organizational levels targeted $[16,17]$. This implies that the measurement of a single outcome on the level of one target group does not sufficiently depict how the intervention causes change and which 
factors influence the outcome. Yet, this knowledge is necessary to adapt interventions to other settings and groups. This means, that in complex interventions, outcomes have to be assessed at all steps of the intervention process and in all groups or participants involved, in order to find out whether and how an intervention will work in practice [17]. If complex interventions do not show the anticipated effects, the process evaluation aims to find the reason for this and how the intervention should be adapted to improve the intended outcomes.

According to the UK Medical Research Council's framework for the development and evaluation of complex interventions, the first question to be answered is as follows: what is the theory behind the intervention? Why is the intervention supposed to work, in which participants and by which mechanisms [18]?

On this theoretical basis, intervention components, participants that are involved and context factors, as well as expected effects, can be depicted in a logic model $[18,19]$.

A logic model allows to allocate outcomes to participants and processes and thus forms the foundation for the development of the research design and the instruments of the process evaluation. Furthermore, contextual factors inherent to participants and organizations are considered, as they influence the procedural and overall outcomes.
The process evaluation aims to depict how the STADPLAN ACP intervention is embedded in the context of participating HCS, patients, and caregivers, how it was received and accepted, and whether it is feasible in the home care setting.

\section{Methods \\ STADPLAN logic model}

We developed and piloted the STADPLAN logic model. Taking necessary adaptations of the intervention to the German home care setting into account, we created and discussed the logic model within the collaborating group (all authors) to ensure that relevant actors and procedural outcomes were incorporated. In the pilot study, we tested the evaluation instruments for feasibility.

The focus of the process evaluation lies on all participants involved and their context, namely the HCS, nurses, patients (or clients) of the HCS and their caregivers (i.e., family caregivers or surrogates of the participating patients).

The logic model for the STADPLAN trial describes (1) the intervention, (2) the implementation, (3) the participants and their mutual relationship, (4) their context, and (5) the anticipated procedural outcomes on the individual level (see Fig. 1).

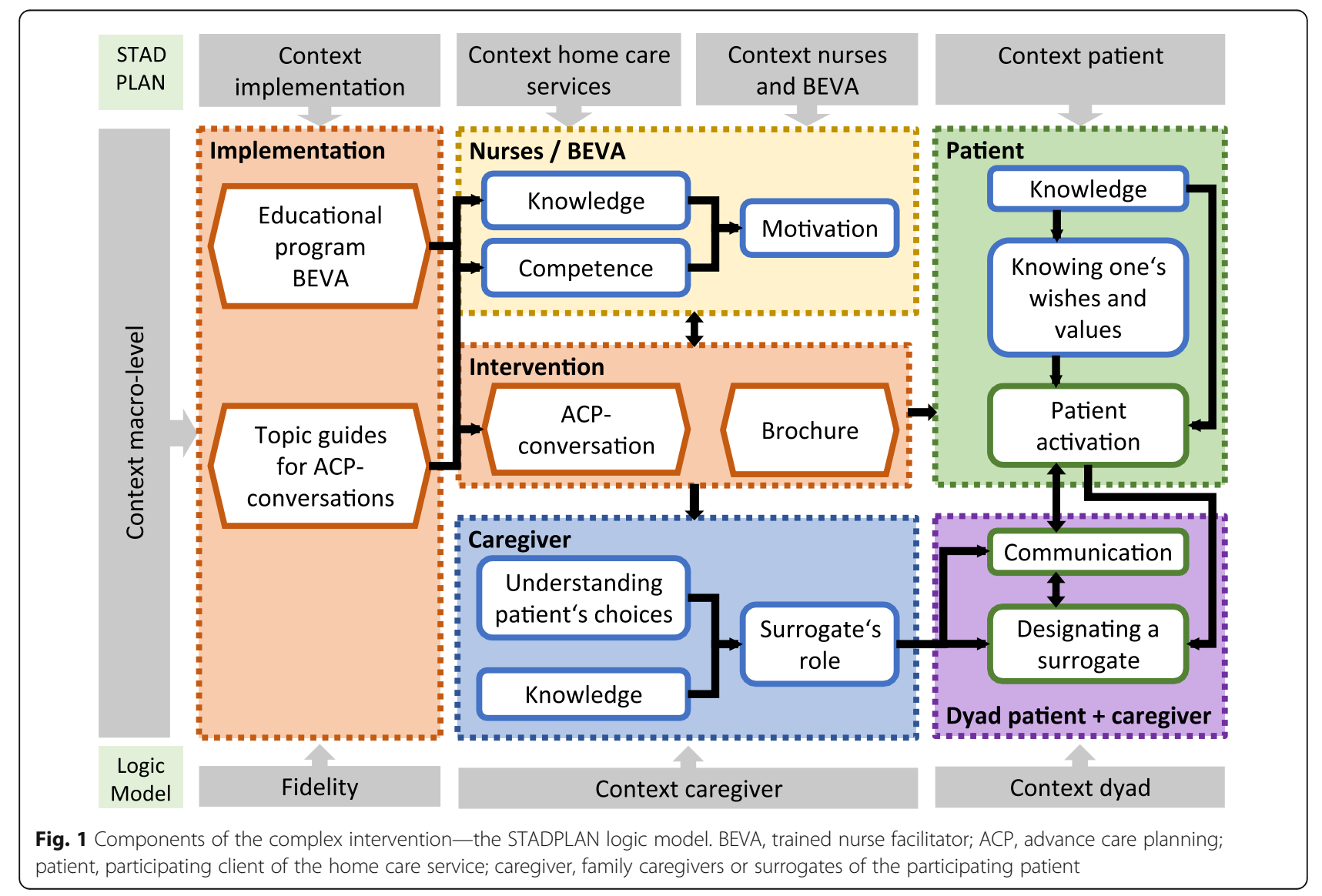




\section{Intervention}

The STADPLAN core intervention consists of two guided ACP conversations with patients and caregivers led by trained nurses (BEVA, German acronym for "facilitator for ACP in the home care setting"), including an information brochure with workbook.

During the first conversation, the BEVAs collect information on patients' ACP activities to date and hand out the information brochure. BEVAs assess patients' knowledge about ACP and provide additional information accordingly. They motivate patients to read the brochure and use the questions and commentary fields to reflect upon their thoughts regarding ACP. They invite patients to include one or more persons of trust, such as family members or designated surrogates (referred to as "caregivers"), to take part in the second conversation.

In this second conversation, BEVAs talk with the patients about their views on life, quality of life, life expectancy, situations of decisional incapability, and wishes regarding life-prolonging treatment.

The focus of the conversation is to support patients in reflecting about their wishes and values, to guide the involved persons of trust in listening to patients' views and in their role as proxy decision maker, and to facilitate further conversation within the family. The aim of the intervention is thus to enable patients to act in their own ACP. The conversations follow structured topic guides, containing commentary fields which the BEVAs use during the conversations to document the discussed topics.

The information brochure with workbook contains information on ACP in plain language and contact details of regional advisory services. The workbook section contains questions facilitating reflection on ACP and room to write them down.

\section{Implementation}

Trained nurses of the participating HCS will conduct the intervention. Their training (educational program) encompasses two 1-day workshops, course material, the information brochure, and topic guides with commentary fields for ACP conversations.

The first workshop provides knowledge on ACP, types of $\mathrm{AD}$ (advance directives), surrogate decision-making, and training on the use of the topic guides, which support BEVAs in leading the conversation and ensure that all relevant issues are discussed. Furthermore, the topic guides serve as documentation for adherence, as BEVAs are instructed to use all commentary fields to document what was discussed.

The second workshop is designed to refresh content of the first workshop and to address BEVAs' experiences with ACP conversations. It is therefore tailored to the BEVAs' needs, based on structured modules. Before we finally determine which modules to address during the workshop, we will question BEVAs via phone about their experiences with the
ACP conversations and suggestions to identify workshop topics. Based on experiences of the pilot study, these could be repetition of informational content; simulation of difficult conversations; peer discussion of experiences regarding recruitment, organization, or challenging interview situations; or other topics requested by the BEVAs. At the end of the workshop day, we will reflect on BEVAs experiences and discuss their overall assessment of the study in a focus group.

\section{Participants}

Participants of the complex intervention are patients, BEVAs, and caregivers. Patients are clients of the participating HCS, 60 years or older, and care dependent (based on assessment by the long-term care insurance). Caregivers are family members who are defined by patients as their main carers. BEVAs are registered nurses of the participating HCS.

\section{Context}

To describe contextual factors is essential in complex interventions, as these can affect the implementation and mechanisms of impact of the intervention, thus potentially influencing the targeted outcomes. Contextual factors differ, depending on the setting of an intervention, and have to be addressed specifically tailored to the intervention and setting to be evaluated [18]. Different context perspectives related to all participants and levels of the core intervention can be determined in the STADPLAN study.

- Context of the HCS: basic characteristics, resources, organizational norms regarding ACP

- Context of nurses and BEVAs: experiences, attitudes, expectations that are relevant for the intervention

- Context of patients and caregivers: experiences, attitudes, expectations, relationship within the dyad and with the HCS

- Context of the implementation and the intervention: organization and conduct of the educational program and ACP conversations

- Context macro-level: actual and perceived norms regarding ACP in the home care setting in general, new developments regarding ACP (like legislation or changes in funding), other incidents affecting the daily practice of HCS with potential influence on the study procedures

\section{Anticipated procedural outcomes}

We will assess procedural outcomes primarily on the individual level, addressing, e.g., knowledge, attitudes, and self-perceived competencies. The educational program enables and motivates nurses to conduct meaningful ACP conversations with patients and their caregivers. Patients learn about ACP and reflect upon what matters to them in life and what this implies for 
end-of-life care. They feel prepared to actively engage in their own ACP. Caregivers gain knowledge on ACP and a better understanding of patients' wishes and attitudes. Thus, they feel reassured in their role as surrogates. The intervention facilitates the designation of a surrogate and the communication on ACP within the patient/caregiver dyad.
Process evaluation framework and methods

The process evaluation follows the course of the main study as depicted in Fig. 2. We chose a mixed methods approach to allow for findings, we did not anticipate emerging, and we will be able to link qualitative with quantitative outcomes, thus enriching our results. Data will be collected at baseline (t0), at the second day of the

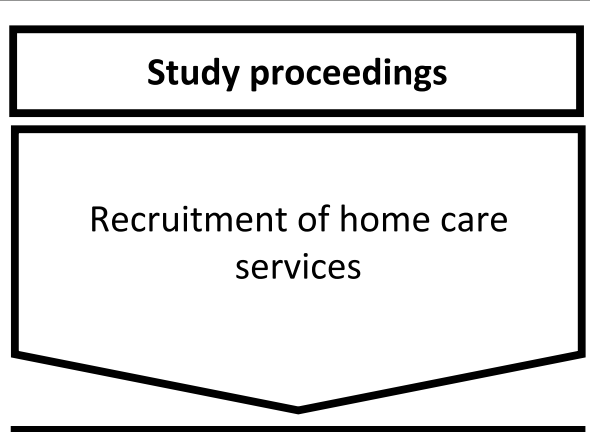

Recruitment of patients
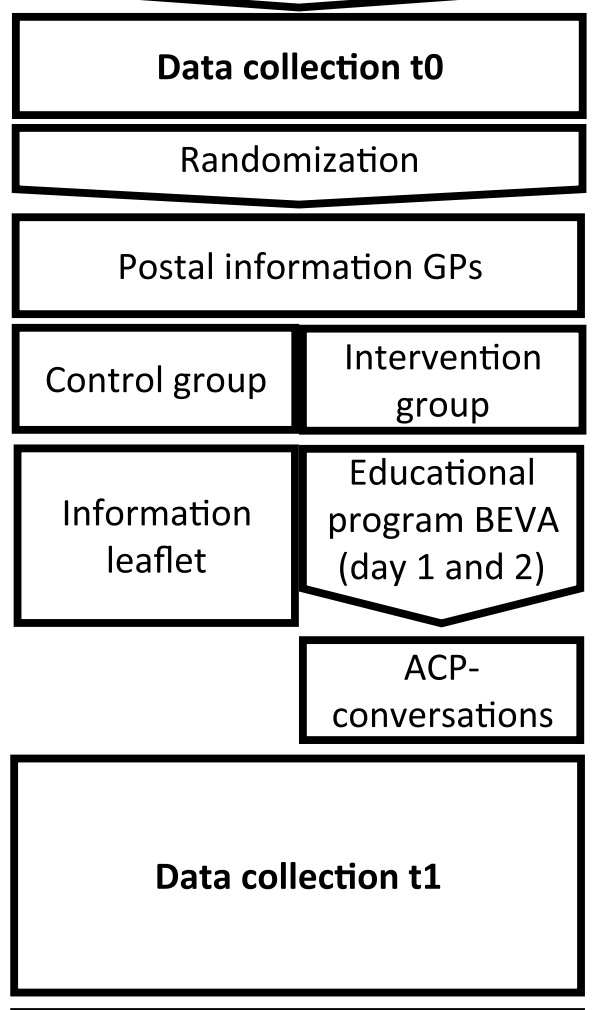

Data collection t2
Process evaluation
Documentation of HCS
recruitment process
Basic characteristics of HCS
Interviews with heads of HCS
Interviews with staff / BEVA

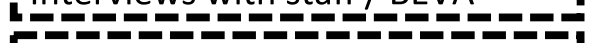
Documentation of recruitment \& Inon-participation (by HCS)

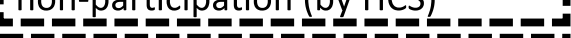
Face-to-face questionnaire with patients

\section{I}

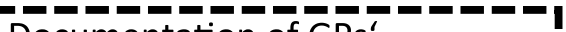

Documentation of Gs'

Information and communication I

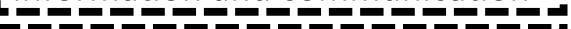
IG: Semi-structured interviews with caregivers

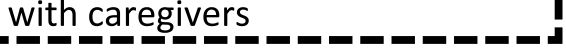
Wh

IG (at educational program): Observation, evaluation form BEVA, focus group with BEVA

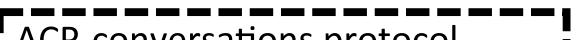
ACP-conversations protocol I (BEVA)

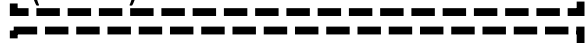
IG: Questionnaire with patients; I semi-structured interviews with caregivers CG: Questionnaire with patients 1

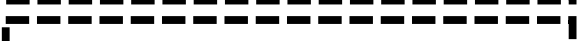
IG, CG: Basic characteristics HCS; interviews with heads of HCS CG: Questionnaire nursing staff CG: Questionnaire nursing staff

Fig. 2 Flowchart of the STADPLAN study and process evaluation. HCS, home care service; GP, general practitioner; BEVA, trained nurse; IG, intervention group; CG, control group; ACP, advance care planning; nursing staff, nurses in the control group who will take part in a 1-day workshop offered to control group HCS 
educational program, and at $\mathrm{t} 1$ (6 months after baseline) and t2 (12 months after baseline) (see Table 1). Baseline is defined on the individual patient's level.>

We will use a variety of instruments to capture intervention processes. The process evaluation is based on the MRC framework on process evaluations for complex interventions [18] as shown in Fig. 3.

\section{Context of the intervention delivery}

To describe the context of the participating HCS, we will use qualitative interviews with the heads of the HCS and nursing staff and assess basic characteristics such as ownership, number of clients, and number and qualification of staff quantitatively [20].

To gauge overall prerequisites for the conduct of our study in the home care setting, we will transparently describe the recruitment process. Time constraints, staff shortage, and scarce funding might hinder HCS to participate in the study and influence the study's progress. We will therefore document non-participation of all invited HCS during the recruitment process in a standardized template at each study site. The HCS will anonymously document the recruitment of participants with standardized forms and question non-participants on patients' level for their reasons to decline. We will categorize reasons for non-participation on HCS and patient level and report them descriptively. Furthermore, we will discuss recruitment obstacles within a focus group on day 2 of the educational program with the present BEVAs.

We will conduct face-to-face interviews with closed and open-ended questions with patients, concerning their knowledge and expectations regarding ACP [21, 22], their engagement in ACP [23], control preferences [24], and their satisfaction with the HCS [25]. The pilot study showed that these data take additional $10-15 \mathrm{~min}$ to collect, which is only feasible with patients who manage the main data collection well. We therefore decided to achieve a convenience sample of 128 patients (4 per cluster).

We will conduct semi-structured interviews with caregivers about their experiences and expectations regarding ACP [26], their satisfaction with the HCS [25], and how they experience their situation as caregiver [27-29]. We aim to recruit a convenience sample of one participant per intervention cluster $(n=16)$ asking patients and caregivers present in the main data collection for participation in a separate telephone interview.

\section{Implementation}

We will focus on four elements of the intervention: the educational program; the recruitment of participants; the ACP conversations; and the information brochure with workbook. We will document the conduct of the educational program by observations focusing on adherence and involvement/interaction of participants. We will base the observations on a template listing each activity of the educational program. The observers (research assistants) will document length and method of the activity, involvement of participants, and questions/ unexpected events. The outcome on BEVAs' level will be assessed with questionnaires at the end of the respective program day, testing knowledge on $\mathrm{ACP}$ and selfreported confidence in communication abilities, and expected feasibility of the upcoming ACP conversations in all participating BEVAs. At the second workshop day of the program, we will conduct a focus group discussion on the experiences of the nurse facilitators, as this will take place after completion of most of the ACP conversations. We aim to include all BEVAs present at the workshop, yet participation is voluntary. We will audiotape the focus group discussion and transcribe verbatim using transcription rules adjusted to the aim of content analysis.

HCS will document recruitment of participants as described above.

We will evaluate the ACP conversations on the level of BEVAs (in the focus group discussion), on the level of patients at $\mathrm{t} 1$ using face-to-face questionnaires with open-ended questions, and on the level of caregivers using qualitative telephone interviews. This multidimensional approach allows for gaining a comprehensive picture of all involved parties' experiences. The same approach will serve to evaluate the information brochure with workbook.

\section{Implementation fidelity}

We will use the documentation of the BEVAs' ACP conversations to estimate adherence to the planned procedure. The topic guide forms provide commentary fields and BEVAs are instructed to document whether the questions were discussed and concerning which content or result. BEVAs' experiences with the topic guides, feasibility, and reasons for non-adherence will also be discussed in the focus groups.

\section{Mechanisms of impact}

Mechanisms of impact describe reciprocal influence and interaction of participants and intervention. In this case, this means the influence of the ACP conversations on patients, caregivers, and BEVAs and how this shapes the conduction of the intervention. We will evaluate these mechanisms at $\mathrm{t} 1$ in patients with open-ended questions regarding their experiences and fulfillment of expectations as well as their perception of BEVAs' performance (face-to-face questionnaire patients, $n=64$, intervention group). We will interview caregivers by phone at t1, focusing on individual experiences, perception of BEVAs' 
Table 1 Process evaluation schedule of enrolment, interventions, and assessments

\begin{tabular}{|c|c|c|c|c|}
\hline & \multicolumn{4}{|c|}{ STUDY PERIOD } \\
\hline & Enrolment & Allocation & $\begin{array}{l}\text { Post- } \\
\text { allocation }\end{array}$ & Close-out \\
\hline TIMEPOINT & $-t_{1}$ & 0 & $\begin{array}{l}t_{1} \text { (6 months } \\
\text { after baseline) }\end{array}$ & $\begin{array}{l}t_{2}(12 \\
\text { months after } \\
\text { baseline) }\end{array}$ \\
\hline \multicolumn{5}{|l|}{ ENROLMENT: } \\
\hline Eligibility screen & $X$ & & & \\
\hline Informed consent & $X$ & & & \\
\hline Allocation & & $X$ & & \\
\hline \multicolumn{5}{|l|}{ INTERVENTIONS: } \\
\hline IG: ACP-Information and conversation & & 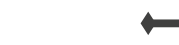 & & $\rightarrow$ \\
\hline Control group: optimized usual care & & 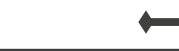 & $\rightarrow$ & \\
\hline \multicolumn{5}{|l|}{ ASSESSMENTS: } \\
\hline $\begin{array}{l}\text { Head of HCS: Experiences with ACP, implementation } \\
\text { of ACP in the HCS, motivation and expectations } \\
\text { regarding study participation }\end{array}$ & $\mathrm{X}$ & & & \\
\hline $\begin{array}{l}\text { Head of HCS: Experiences with ACP, implementation } \\
\text { of } A C P \text { in the HCS, experiences within the study and } \\
\text { further plans reg. ACP }\end{array}$ & & & & X \\
\hline $\begin{array}{l}\text { Basic data HCS: ownership, number of clients, } \\
\text { qualification and number of staff, ACP related services }\end{array}$ & X & & & $X$ \\
\hline $\begin{array}{l}\text { Nursing staff pre allocation: Formal qualification, work } \\
\text { experience, experiences with ACP, motivation, and } \\
\text { expectation regarding study participation }\end{array}$ & $\mathrm{X}$ & & & \\
\hline $\begin{array}{l}\text { BEVA: experiences with the intervention and study } \\
\text { participation in general }\end{array}$ & & & X & \\
\hline $\begin{array}{l}\text { Control group nursing staff: experiences during the } \\
\text { course of the study }\end{array}$ & & & & $\mathrm{X}$ \\
\hline $\begin{array}{l}\text { Patients (IG and CG): Experiences and expectations } \\
\text { regarding ACP and study participation, ACP } \\
\text { knowledge, control preferences, satisfaction with HCS }\end{array}$ & $\mathrm{X}$ & & & \\
\hline $\begin{array}{l}\text { Patients (IG): Experiences regarding ACP and study } \\
\text { participation, ACP knowledge, control preferences, } \\
\text { satisfaction with HCS, satisfaction with ACP } \\
\text { conversations }\end{array}$ & & & $\mathrm{X}$ & \\
\hline $\begin{array}{l}\text { Patients (CG): Experiences regarding ACP and study } \\
\text { participation, ACP knowledge, control preferences, } \\
\text { satisfaction with HCS }\end{array}$ & & & $\mathrm{X}$ & \\
\hline $\begin{array}{l}\text { Family caregivers (IG): Experiences and expectations } \\
\text { regarding ACP and study participation, ACP } \\
\text { knowledge, caregiver burden, satisfaction with HCS }\end{array}$ & $\mathrm{X}$ & & $\mathrm{X}$ & \\
\hline
\end{tabular}

$A C P$ advance care planning, optimized usual care control group participants receive short written information on $A C P, H C S$ health care service, $B E V A$ trained nurse facilitator for ACP conversations, IG intervention group, CG control group 


\begin{tabular}{|c|c|}
\hline \multicolumn{2}{|c|}{$\begin{array}{l}\text { A) Identify context of intervention delivery: } \\
\text { Context of participating Home Care Services (HCS) and BEVAs: } \\
\text { - Semi-structured interviews with heads of HCS } \\
\text { - Semi-structured interviews with nursing staff / BEVA } \\
\text { - Questionnaire: basic characteristics and services of HCS } \\
\text { - Documentation of the recruitment process of HCS }\end{array}$} \\
\hline $\begin{array}{l}\text { B) Assess impler } \\
\text { Educational } \\
\text { program BEVA } \\
\text { Describe program } \\
\text { theory }\end{array}$ & $\begin{array}{l}\text { Observation } \\
\text { Questionnaire BEVA (post) } \\
\text { Focus group BEVA (day 2) } \\
\text { Documentation of recruitment } \\
\text { Focus group BEVA } \\
\text { Focus group BEVA } \\
\text { Face-to-face questionnaire patients } \\
\text { Semi-structured interviews caregivers } \\
\text { Semi-structured interviews caregivers } \\
\text { Face-to-face questionnaire patients } \\
\text { Focus group BEVA } \\
\text { ACP-conversations protocol } \\
\text { Focus group BEVA }\end{array}$ \\
\hline
\end{tabular}

performance, and perceived benefits or pitfalls of the intervention ( $n=16$, intervention group). We will assess impact on the communication about ACP in the family and ACP engagement in all patients as part of the main data collection at $\mathrm{t} 0-\mathrm{t} 2$. In the semi-structured interviews, we will ask caregivers about habits of decisionmaking within the dyad at $\mathrm{t} 0$ and $\mathrm{t} 1$.

\section{Recruitment and data collection}

We aim to recruit patients' caregivers or family members, heads of HCS, and BEVAs/nursing staff of the control group, related to all HCS (see Table 2, recruitment goals). We will obtain written consent and interview patients in conjunction with the main data collection visits at their homes. We will interview heads of HCS and nursing staff/BEVA face-to-face or by phone. At $\mathrm{t} 1$, we will perform group-specific questionnaires with patients and interviews with caregivers separately via phone. Informed consent will be sought from all participants and documented. Experiences from the pilot study indicated that recruitment would take several months. Therefore, baseline has been defined on individual participants' level. T1 and t2 take place 6 and 12 months after baseline.

We will conduct semi-structured interviews face-toface or by phone and audiotape and transcribe interviews verbatim, anonymizing data in the transcription process. We will store all data for the process evaluation in an anonymized manner, and document only study site and date/measurement point on data sheets, as we decided against linking the process data and the main outcome on an individual level and opted for a cumulated

Table 2 Recruitment goals

\begin{tabular}{lllll}
\hline Participant & $\boldsymbol{N}$ at t0 & $\boldsymbol{N}$ at t1 & $\boldsymbol{N}$ at t2 & Method \\
\hline $\begin{array}{l}\text { Head of HCS } \\
\text { BEVA/control group nursing staff }\end{array}$ & 32 & 32 & Semi-structured interview \\
$\begin{array}{l}\text { BEVA (IG) } \\
\text { Nursing staff (CG) }\end{array}$ & 3 & & Semi-structured interview \\
$\begin{array}{l}\text { Patients (IG + CG) } \\
\text { Patients (IG) }\end{array}$ & 128 & 16 & Focus group (t1/day 2 workshop, one per study site) \\
$\begin{array}{l}\text { Patients (CG) } \\
\text { Family caregivers (IG) }\end{array}$ & 64 & & Questionnaire (telephone) \\
\hline
\end{tabular}

HCS home care service, BEVA trained nurse, IG intervention group, CG control group 
comparison between the points of assessment at baseline/enrolment and $\mathrm{t} 1$, and between control group and intervention group. We will use data necessary to contact patients and family caregivers at home only to access them for scheduling and conducting data collection. During our pilot study, we tested the interviews with caregivers $(n=5)$, patients $(n=5)$, heads of HCS $(n=4)$, and staff $(n=3)$. Only minor revisions were necessary where we deemed questions redundant.

\section{Data analysis}

We will analyze data from all semi-structured interviews using the program MAXQDA Standard 12 (Release 12.3.5, VERBI GmbH Berlin). We will conduct a content analysis and use a deductive-inductive approach [30]. Based on the constructs of the logic model, we will code the data, allowing new findings to emerge and to be integrated. By summarizing the perspectives of the different participants on the level of the logic model as coding scheme, we will be able to depict all constructs from several points of view. We will analyze quantitative data descriptively using IBM SPSS Statistics (Version 22.0.0.1) and integrate findings into the structure of the qualitative analysis. We developed an analysis plan, associating the qualitative and quantitative variables with the constructs of the logic model. We will provide a codebook in which the variables of the mixed methods are linked to the codes.

\section{Ethics}

ACP requires reflecting on personal values, end-of-life care, and death. These topics must be treated in a sensitive and respectful manner, as they may cause distress in participants. Data collection, especially semi-structured interviews, will be conducted by experienced research assistants, who are not involved in patients' care and have no professional or personal relationship with the patient. Interviews with patients will be conducted at their own homes in familiar environment. Patients will receive written information about the study and will have the possibility to ask questions before giving written consent. They will be informed about the possibility to end participation at any time without negative consequences regarding their care or relationship towards the HCS. Heads and BEVAs of the collaborating HCS and caregivers will receive oral information on the aim of the data collection, anonymization, data storage, dissemination, and voluntary consent. The have the possibility to ask questions and give oral consent or decline without negative consequences.

\section{Trial status}

Protocol version: Version 3 - 29.12.2019.
The STADPLAN Trial is currently being implemented and will be completed in January 2021. Recruitment started on May 29, 2019, and will be completed by January $31,2020$.

\section{Discussion}

The STADPLAN study is a large cluster-randomized controlled trial and one of the first trials in ACP in community dwellers. It will provide evidence on the effectiveness of an ACP intervention provided by trained nurses in home care. The comprehensive process evaluation will indicate how the ACP intervention is related to patient activation, ACP engagement, and surrogate designation and evaluate its feasibility in the home care setting. We will follow current internationally acknowledged guidelines [18]; the process evaluation is founded on a theory-based logic model. Yet, it will not be possible to illuminate every process in detail. For example, we will use the BEVAs' documentation to estimate how closely the planned procedure in the ACP conversations was followed and ask participants for their experiences. These surrogate parameters will indicate to which degree the intervention was implemented as intended. We refrained from observing or audiotaping the conversations, as ACP is a sensitive topic, the conversations should be confidential, and participants (including patients, caregivers, and BEVAs) need to feel comfortable. Patients show varying levels of cognitive capacity and exhaustibility, so we decided to use a convenience sample, taking patients' individual conditions into account and asking for consent to conduct extra questionnaires. This might introduce a selection bias, e.g., for ACP knowledge, or satisfaction with the HCS, but we chose to accept this rather than losing participants during data collection. Therefore, we pre-tested instruments and processes which have proven to be feasible in elderly participants.

Process evaluation is of utmost importance, but still it must be feasible. The MRC framework recommends to rather reduce the amount of data collected in favor of collecting the right data. For this reason, we developed the logic model within the project group, which provides guidance and commitment on the relevant questions.

Looking at the key recommendations for process evaluation by the MRC framework [18], the strengths of the STADPLAN process evaluation can be described accordingly:

\section{Planning of the process evaluation}

For this phase, the key recommendations focus on the relationship, the expertise, and the synchronization of the process and outcome evaluation teams. 
Researchers of four universities with experiences in successful earlier study collaborations conduct the STADPLAN study. We developed the intervention and the process evaluation in close cooperation but with different responsibility among partners. All partners discussed the logic model intensively, to make sure that it represents the linking key.

Thus, the close relationship, yet distinct responsibility for intervention development and process evaluation as required according to the MRC framework, as well as the necessary expertise, is fulfilled in this study.

\section{Design and conduct}

Key recommendations regarding this phase are as follows: describing the intervention and clarifying causal assumptions, defining the most important research questions, and selecting a combination of methods. In the STADPLAN study, we use a logic model to show the main elements of the intervention and the procedural outcomes we focus on. To define the relevant outcomes, we considered previous research in this domain. Furthermore, we approached important stakeholders in advance, both on local and national levels, for example, the association of legal guardians and the federal nursing council. The advisory board incorporates members representing research in ACP, geriatrics, and primary care as well as a patients' organization. Thus, we were able to collect expertise and receive support on relevant contextual factors and current considerations in the research field.

The instruments are based on theoretical foundation and the recommendations of stakeholders and target each step of the MRC evaluation framework (see Fig. 3). We will use a mixed methods approach of qualitative and quantitative methods including all defined participants of the intervention and their context. We will collect part of the data in the whole sample (such as assessing the ACP conversations protocol or interviews with the heads of HCS); part will be collected in a subsample. The approach has been successfully applied in previous process evaluation designs [31-33].

\section{Analysis}

Key recommendations of this phase are considering mixed methods data, analyzing these in an iterative process, building qualitative and quantitative analyses on each other, and publishing process data early in advance to main outcome measures. In our pilot study, we already developed the analysis scheme for the qualitative data, building a deductive analysis process upon the concepts incorporated in our logic model.

This allows us to analyze the process data alongside the running trial. We decided to set data collection points for process evaluation in BEVAs, patients, and caregivers around $\mathrm{t} 1$ (6 months after baseline), after implementation of the intervention. This will facilitate the timely completion of the process evaluation. At the end of the study, we will conduct interviews with the heads of the HCS for a full account of their experiences.

\section{Reporting}

We will disseminate results via conferences, scientific forums, and publications as well as via reports and workshops targeted towards stakeholders. In reporting this protocol and the results of the process evaluation, we followed the MRC framework and previous study protocols and process evaluations with comparable approaches $[34,35]$. In addition, the complex intervention is reported based on the Criteria for Reporting the Development and Evaluation of Complex Interventions (CReDECI 2) [36] (Additional file 1).

After completion of the study, we will invite all stakeholders for a presentation of first results by the universities on a local level, promoting discussion and networking. Thus, we aim to enhance impact of research on the local level of nursing practice.

\section{Supplementary information}

Supplementary information accompanies this paper at https://doi.org/10. 1186/s13063-020-04529-2.

Additional file 1. CReDICI 2 checklist STADPLAN project.

\section{Abbreviations}

ACP: Advance care planning: BEVA: Facilitator for ACP in the home care setting (German acronym: Begleiterin für vorausschauende Versorgungsplanung in der Ambulanten Pflege); CG: Control group; CReDECl: Criteria for the Reporting of the Development and Evaluation of Complex Interventions; GP: General practitioner; HCS: Home care service; IG: Intervention group; MRC: Medical Research Council; PAM: Patient Activation Measure; STADPLAN: Study on advance care planning in caredependent community dwelling older persons

\section{Acknowledgements}

We would like to thank the members of the advisory board for helpful advice regarding the used measurement instruments and practical aspects for conducting the STADPLAN intervention (especially Jürgen in der Schmitten, Michael Freitag, Jürgen M. Bauer, and Brigitte Bührlen). We also thank Professor Katrin Balzer, University of Lübeck, for providing valuable advice on planning and conducting the process evaluation.

\section{Authors' contributions}

KS and SK developed the design of the current study; RS, AB, ÄK, HL, JK-N, $\mathrm{GM}$, and FH discussed and substantially revised the work during the development. All authors read and approved the submitted version and have agreed both to be personally accountable for their own contributions and to ensure that questions related to the accuracy or integrity of any part of the work, even ones in which they were not personally involved, are appropriately investigated, resolved, and the resolution documented in the literature.

\section{Funding}

The study is funded by the German Federal Ministry of Education and Research (BMBF grant 01GL1707A-D). Funders will not interfere at any stage of the study design and research process. 


\section{Availability of data and materials}

The datasets generated and analyzed during the current study are not publicly available due to conditions of informed consent with participants. Please contact corresponding author in case of reasonable requests.

\section{Ethics approval and consent to participate}

Ethical approval for the process evaluation was obtained in conjunction with the main study by the ethics committees of the participating universities (Oldenburg: Ref.-No. 2019-024, Halle (Saale): Ref.-No. 2019-045, Lübeck: Ref.No. 19-080). Informed content to participate was obtained of all participants as stated above.

\section{Consent for publication}

Not applicable.

\section{Competing interests}

The authors declare that they have no competing interests.

\section{Author details}

${ }^{1}$ Institute for Social Medicine and Epidemiology, Nursing Research Unit, University of Lübeck, Ratzeburger Allee 160, 23562 Lübeck, Germany. ${ }^{2}$ Department for Health Services Research, Faculty of Medicine and Health Sciences, Carl von Ossietzky University Oldenburg, Ammerländer Heerstraße 140, 26129 Oldenburg, Germany. ${ }^{3}$ Medical Faculty, Institute for Health- and Nursing Science, Martin Luther University Halle-Wittenberg, Magdeburger Straße 8, 06112 Halle (Saale), Germany. ${ }^{4}$ Center for Health Economics and Health Services Research, Schumpeter School of Business and Economics, University of Wuppertal, Rainer-Gruenter-Straße 21, 42119 Wuppertal, Germany. ${ }^{5}$ Institute for Nursing Science, University of Cologne, Gleueler Straße 176 - 178, 50935 Köln, Germany.

Received: 29 December 2019 Accepted: 18 June 2020 Published online: 16 July 2020

\section{References}

1. Sudore RL, Lum HD, You JJ, Hanson LC, Meier DE, Pantilat SZ, et al. Defining advance care planning for adults: a consensus definition from a multidisciplinary delphi panel. J Pain Symptom Manag. 2017;53(5):821-32.e1.

2. Zwakman M, Jabbarian $\mathrm{L}$, van Delden J, van der Heide A, Korfage IJ, Pollock K, et al. Advance care planning: a systematic review about experiences of patients with a life-threatening or life-limiting illness. Palliat Med. 2018;32(8):1305-21 Epub 2018/06/30.

3. Jabbarian $L$, Zwakman $M$, van der Heide A, Kars MC, Janssen DJA, van Delden JJ, et al. Advance care planning for patients with chronic respiratory diseases: a systematic review of preferences and practices. Thorax. 2018; 73(3):222-30 Epub 2017/11/08.

4. Glaudemans JJ, Moll van Charante EP, Willems DL. Advance care planning in primary care, only for severely ill patients? A structured review. Fam Pract. 2015:32(1):16-26 Epub 2014/11/09.

5. Koch KA. Patient Self-Determination Act. J Fla Med Assoc. 1992;79(4):240-3.

6. Johnston C, Liddle J. The Mental Capacity Act 2005: a new framework for healthcare decision making. J Med Ethics. 2007;33(2):94-7.

7. Wiesing $\mathrm{U}$, Jox RJ, Hessler HJ, Borasio GD. A new law on advance directives in Germany. J Med Ethics. 2010;36(12):779-83 Epub 2010/11/30.

8. Hammes BJ, Rooney BL, Gundrum JD. A comparative, retrospective, observational study of the prevalence, availability, and specificity of advance care plans in a county that implemented an advance care planning microsystem. J Am Geriatr Soc. 2010;58(7):1249-55 Epub 2010/07/24.

9. In der Schmitten J, Lex K, Mellert C, Rotharmel S, Wegscheider K, Marckmann G. Implementing an advance care planning program in German nursing homes: results of an inter-regionally controlled intervention trial. Dtsch Arztebl Int. 2014;111(4):50-7 Epub 2014/03/13.

10. Sommer S, Marckmann G, Pentzek M, Wegscheider K, Abholz HH, in der Schmitten J. Advance directives in nursing homes: prevalence, validity, significance, and nursing staff adherence. Dtsch Arztebl Int. 2012;109(37): 577-83.

11. Schnakenberg R, Silies K, Berg A, Kirchner A, Langner H, Chuvayaran Y, et al. Study on advance care planning in care dependent community-dwelling older persons in Germany (STADPLAN): protocol of a cluster-randomised controlled trial. BMC Geriatr. 2020;20(1):142 Epub 2020/04/19.
12. Hammes BJ, Rooney BL. Death and end-of-life planning in one midwestern community. Arch Intern Med. 1998;158(4):383-90 Epub 1998/03/06.

13. Hammes BJ, Briggs L. Building a systems approach to advance care planning: bereavement and advance care planning services: Gundersen Lutheran Medical Foundation, Incorporated; 2011.

14. Hibbard JH, Mahoney ER, Stockard J, Tusler M. Development and testing of a short form of the patient activation measure. Health Serv Res. 2005;40(6 Pt 1):1918-30 Epub 2005/12/13.

15. Zill JM, Dwinger S, Kriston L, Rohenkohl A, Härter M, Dirmaier J. Psychometric evaluation of the German version of the patient activation measure (PAM13). BMC Public Health. 2013;13(1):1027.

16. Craig P, Dieppe P, Macintyre S, Michie S, Nazareth I, Petticrew M. Developing and evaluating complex interventions: the new Medical Research Council guidance. Int J Nurs Stud. 2013;50(5):587-92 Epub 2012/11/20.

17. Craig P, Dieppe P, Macintyre S, Michie S, Nazareth I, Petticrew M. Developing and evaluating complex interventions: new guidance. London: Medical Research Council; 2013.

18. Moore GF, Audrey S, Barker M, Bond L, Bonell C, Hardeman W, et al. Process evaluation of complex interventions: Medical Research Council guidance. BMJ. 2015;350:h1258 Epub 2015/03/21.

19. Kellog W. Using logic models to bring together planning, evaluation, and action: logic model development guide. Kellog Foundation: Michigan; 2004.

20. Pflegestatistik 2017 - Pflege im Rahmen der Pflegeversicherung. Statistisches Bundesamt Destatis; 2018 [05.11.2019]; Available from: https://www.destatis.de/DE/Themen/Gesellschaft-Umwelt/Gesundheit/ Pflege/_inhalt.html.

21. Evans N, Bausewein C, Menaca A, Andrew EV, Higginson IJ, Harding R, et al. A critical review of advance directives in Germany: attitudes, use and healthcare professionals' compliance. Patient Educ Couns. 2012;87(3):277-88 Epub 2011/11/26.

22. Sahm S, Will R, Hommel G. Attitudes towards and barriers to writing advance directives amongst cancer patients, healthy controls, and medical staff. J Med Ethics. 2005;31(8):437-40 Epub 2005/08/04.

23. Sudore RL, Heyland DK, Barnes DE, Howard M, Fassbender K, Robinson CA, et al. Measuring advance care planning: optimizing the advance care planning engagement survey. J Pain Symptom Manag. 2017;53(4):669-81.e8 Epub 2017/01/04.

24. Degner LF, Sloan JA, Venkatesh P. The control preferences scale. 1997. 1997. Epub 1997-04-13.

25. Wagner D, Bear M. Patient satisfaction with nursing care: a concept analysis within a nursing framework. J Adv Nurs. 2009;65(3):692-701.

26. Mignani V, Ingravallo F, Mariani E, Chattat R. Perspectives of older people living in long-term care facilities and of their family members toward advance care planning discussions: a systematic review and thematic synthesis. Clin Interv Aging. 2017;12:475-84 Epub 2017/04/21.

27. Stromberg A, Luttik ML. Burden of caring: risks and consequences imposed on caregivers of those living and dying with advanced heart failure. Curr Opin Support Palliat Care. 2015;9(1):26-30 Epub 2015/01/15.

28. Grässel E. Häusliche-Pflege-Skala HPS: zur Erfassung der subjektiven Belastung bei betreuenden oder pflegenden Personen: Vless Verlag; 2001.

29. Graessel E, Berth H, Lichte T, Grau H. Subjective caregiver burden: validity of the 10-item short version of the Burden Scale for Family Caregivers BSFC-s. BMC Geriatr. 2014;14(1):23.

30. Mayring P. Qualitative Inhaltsanalyse. Grundlagen und Techniken. 11., aktualisierte und überarbeitete Auflage ed. Beltz Verlag: Weinheim und Basel; 2010.

31. Richter C, Berg A, Langner H, Meyer G, Kopke S, Balzer K, et al. Effect of person-centred care on antipsychotic drug use in nursing homes (EPCentCare): a cluster-randomised controlled trial. Age Ageing. 2019;48(3): 419-25 Epub 2019/02/27.

32. Abraham J, Kupfer R, Behncke A, Berger-Hoger B, Icks A, Haastert B, et al. Implementation of a multicomponent intervention to prevent physical restraints in nursing homes (IMPRINT): a pragmatic cluster randomized controlled trial. Int J Nurs Stud. 2019;96:27-34 Epub 2019/04/25.

33. Muller M, Bartoszek G, Beutner K, Klingshirn H, Saal S, Stephan AJ, et al. Developing and piloting a multifactorial intervention to address participation and quality of life in nursing home residents with joint contractures (JointConlmprove): study protocol. Ger Med Sci. 2015;13:Doc13 Epub 2015/07/22.

34. Haynes A, Brennan S, Carter S, O'Connor D, Schneider CH, Turner T, et al. Protocol for the process evaluation of a complex intervention designed to 
increase the use of research in health policy and program organisations (the SPIRIT study). Implement Sci. 2014;9:113 Epub 2014/11/22.

35. Boeckmann M, Nohavova I, Dogar O, Kralikova E, Pankova A, Zvolska K, et al. Protocol for the mixed-methods process and context evaluation of the TB \&

Tobacco randomised controlled trial in Bangladesh and Pakistan: a hybrid effectiveness-implementation study. BMJ Open. 2018;8(3):e019878 Epub 2018/04/01

36. Mohler R, Kopke S, Meyer G. Criteria for reporting the development and evaluation of complex interventions in healthcare: revised guideline (CReDECl 2). Trials. 2015;16:204 Epub 2015/05/04.

\section{Publisher's Note}

Springer Nature remains neutral with regard to jurisdictional claims in published maps and institutional affiliations.

\section{Ready to submit your research? Choose BMC and benefit from:}

- fast, convenient online submission

- thorough peer review by experienced researchers in your field

- rapid publication on acceptance

- support for research data, including large and complex data types

- gold Open Access which fosters wider collaboration and increased citations

- maximum visibility for your research: over $100 \mathrm{M}$ website views per year

At $\mathrm{BMC}$, research is always in progress.

Learn more biomedcentral.com/submissions 